\title{
A Guide to Healthy Snacking for the Frail Older Adult ${ }^{1}$
}

\author{
Paula G. Harris-Swiatko and Wendy J. Dahl ${ }^{2}$
}

The frail older adult is a special group consisting of older adults who suffer from disabilities or illnesses that may affect their ability to function in everyday life. Many have difficulty performing and completing simple tasks and require the assistance of a caregiver. Among other challenges they may face on a daily basis, preparing and consuming a healthy diet can be difficult for this group. If frail older adults have trouble preparing and consuming a healthy diet and meeting their nutritional needs, malnutrition may become a concern.

The frail older adult faces unique challenges that may interfere with meeting their nutritional needs. For example, the frail older adult may have problems chewing and swallowing. They may also have gastrointestinal issues, such as heartburn and loss of appetite, and may experience discomfort when eating certain foods. Weakness from bone and muscle loss may also further impair their ability to complete physical tasks, such as cooking. Challenges like these may lead to discouragement, decreased food consumption, and irregular eating patterns, which can further lead to various nutrient deficiencies. In order to help this population overcome some of the challenges of preparing and eating a nutritious diet, caregivers need access to ideas and tools that will help them provide adequate nutrition to the frail older adult. Simple, nutritious snacks that are easy to eat are a great way to add nutritious foods to their diet.

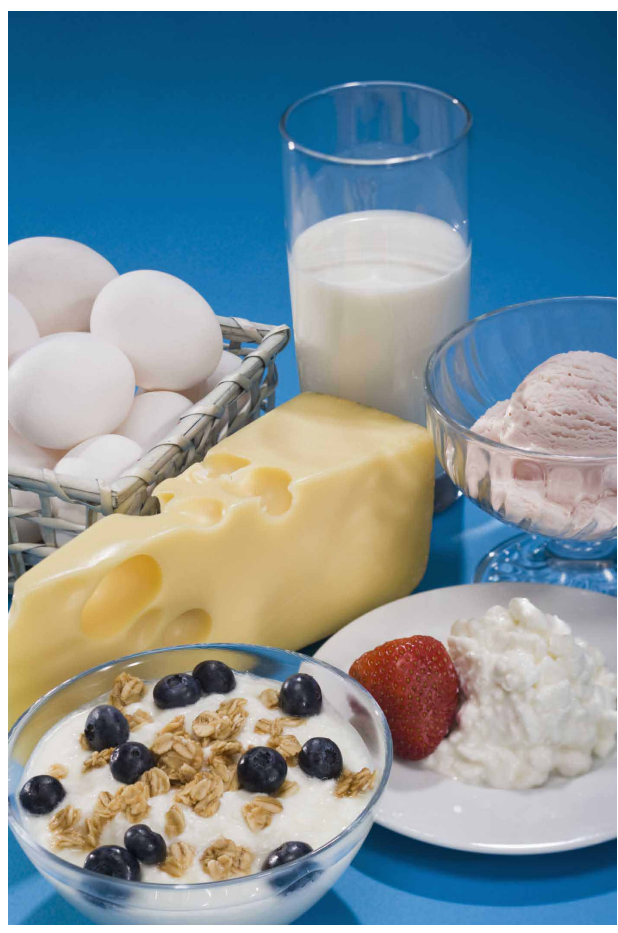

\section{Common Conditions and Diseases of the Frail Older Adult}

The frail older adult faces special challenges from agerelated conditions and diseases that may decrease their quality of life and make preparing and consuming a healthy diet difficult. Most often, the frail older adult who is able to live at home needs the assistance of a caregiver with everyday tasks like preparing meals. Common age-related

1. This document is FSHN12-19, one of a series of the Food Science and Human Nutrition Department, Florida Cooperative Extension Service, Institute of Food and Agricultural Sciences, University of Florida. Original publication date March 2013. Visit the EDIS website at http://edis.ifas.ufl.edu.

2. Paula G. Harris-Swiatko, MS, and Wendy J. Dahl, assistant professor, Food Science and Human Nutrition Department, Institute of Food and Agricultural Sciences, University of Florida, Gainesville, FL 32611. 
illnesses that may cause challenges in daily tasks include the following:

- Frailty syndrome develops because of muscle and bone loss during aging. Frailty syndrome impacts one's ability to complete everyday tasks, such as cooking.

- Muscle weakness commonly develops as a result of aging. Muscle weakness often leads to a decrease in activity and increased frailty because one does not have the strength to do the things that one used to do.

- Osteoporosis is an age-related disease of the bones in which the bones become weak and fragile. Weak bones lead to increased risk of falls and fractures. Many times, broken bones result in lengthy hospital stays. This is a risk to the patient's health because a prolonged hospital stay may weaken the immune system and lessen one's chance of recovery.

\section{Nutrition for the Frail Older Adult}

As people age, their nutrient needs change. Certain vitamins and nutrients become increasingly important, such as calcium and Vitamin D for bone health and protein for healing and muscle health. Malnutrition is more likely to occur in the homebound, frail older adult than in the healthy older adult because the homebound older adult often has more difficulty accessing healthy foods or grocery stores. They are commonly homebound because of disability or illness, and these conditions make preparing meals a challenge. To achieve optimal health, it is important to consume a variety of foods from all of the food groups, which helps to ensure that one gets the nutrients one needs. Consuming snacks that provide key nutrients and taste good is a simple and important way to provide the frail elderly adult with nutrients that are critical to their health.

\section{Protein Needs}

Protein is important for all aging individuals, but it is especially important for the frail older adult. Protein has many functions. It repairs cells, maintains and replaces tissues, and manufactures hemoglobin (red blood cells that carry oxygen). It is essential to blood clotting. It also produces antibodies to fight diseases and illness. Good sources of protein include meat, poultry, fish, dairy products, and legumes.

\section{Calcium and Vitamin D}

Although all vitamins and minerals are necessary for optimal health, calcium and vitamin $\mathrm{D}$ are very important

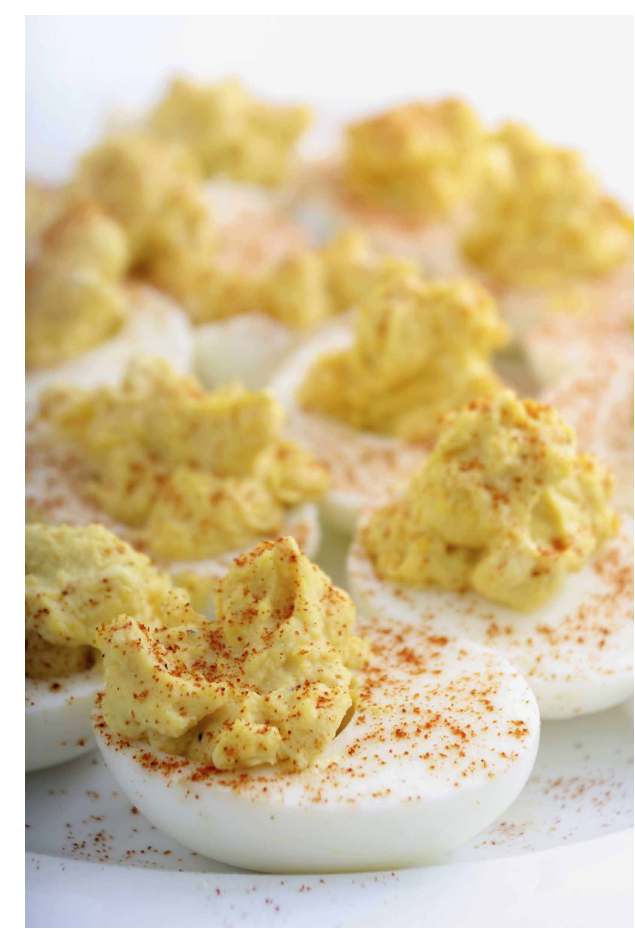

for the frail older adult. Adequate calcium and vitamin D are needed to maintain bones. More vitamin $\mathrm{D}$ is needed from the diet as skin synthesis declines with age. There are ways to add more calcium and vitamin $\mathrm{D}$ to the diet. Dairy foods, particularly milk, yogurt, and cheese, are high in calcium. Other good sources include calcium-fortified versions of orange juice and soy beverages. Food sources of vitamin $\mathrm{D}$ include fatty fish, milk, some yogurts, liver, and egg yolk, as well as fortified breads and cereal. However, many breads and cereals do not have added vitamin $\mathrm{D}$, so it is important to check the label.

\section{Easy Snack Ideas}

Making wise snack choices helps to ensure that frail older adults meet their nutrient needs. Here are some simple, nutritious snacks that are appropriate for the frail older adult. They are easy to chew and swallow as well as easy to prepare, and they taste good! When planning snacks for the frail older adult, it is important to incorporate a variety of healthy food choices. This ensures that snacks are providing a balance of nutrients.

- Deviled eggs: Deviled eggs are a great source of protein. Start by boiling the eggs until hard (ten minutes). Drain the eggs and then cool with cold water or refrigerate them. Place the eggs on a clean cutting board. Remove the shells and slice the eggs in half lengthwise. Remove the yolk with a spoon and place the yolks in a dish. Add mayonnaise to the yolks and stir until moist and creamy. 


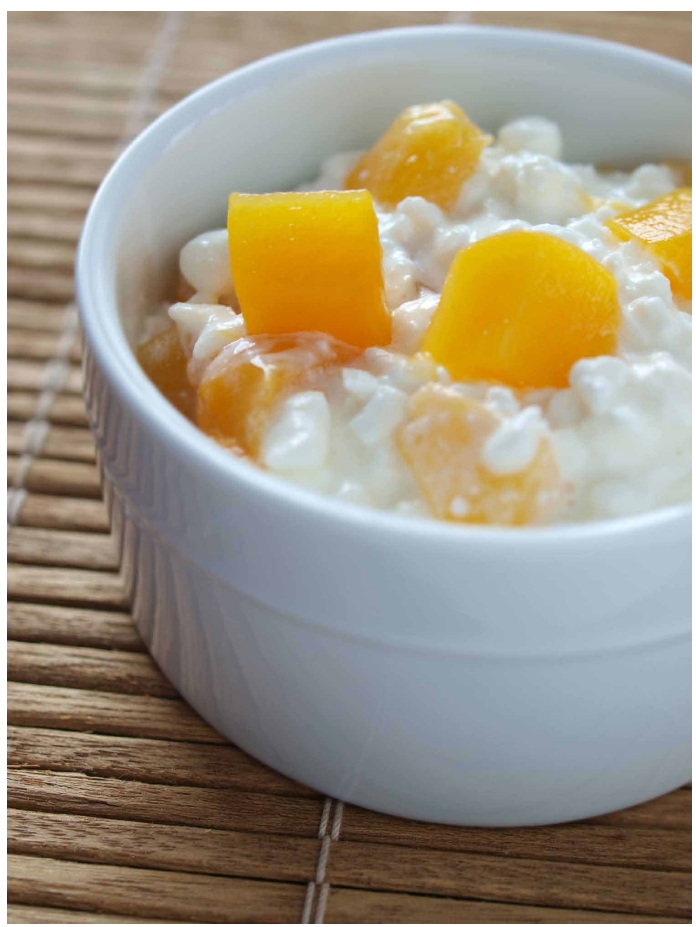

Spoon the yolk mixture into the egg hollows and sprinkle with paprika. Serve chilled.

- Cottage cheese with canned fruit: Cottage cheese is a snack that is very soft and easy to eat. It also provides a good source of protein. Canned pears or peaches with light syrup are tasty and give the cottage cheese some extra flavor. Open the canned fruit and drain. Next, slice the fruit into bite-sized pieces and combine with a few tablespoons of cottage cheese. Mix well and serve chilled.

- Flavored milk: Flavored milks, such as chocolate or strawberry, provide protein, calcium, vitamin $\mathrm{D}$, and energy and are a quick, easy, and tasty snack. Shelf-stable, single-serving versions are particularly convenient.

- Yogurt with fruit: Yogurt is a good snack option for the older adult and provides a source of protein and calcium. Serve half a cup of plain or flavored yogurt topped with fresh berries

- Milk pudding: Pudding is a tasty and convenient snack to keep on hand. Pudding can be bought as shelf-stable, individual servings or as fresh options that require refrigeration. In addition to the commonly available flavors, such as vanilla, chocolate, and butterscotch, traditional favorites of the older adult, rice and tapioca, are available.

- Blended smoothies: Smoothies are simple to prepare with lots of flavors and ingredients to choose from. A basic smoothie starts by pouring a cup of milk into a blender. Add $1 / 2$ cup of yogurt or ice cream and fresh fruit. You can add any fruit you'd like, but try to choose fruits that blend well, like bananas or berries. Drizzle honey on top to add some sweetness. Blend on high until smooth. Pour and serve.

- Cheese: Cheese is tasty and simple to prepare. Block cheddar, Swiss, and mozzarella cheese are good options, but also try the many varieties of specialty cheeses. Cut the cheese into bite-sized cubes or purchase packaged cheeses that are already cubed.

- Ice cream with strawberry drizzle: Ice cream is a delicious treat that most people simply cannot resist. Ice cream comes in a variety of flavors and also in differing fat contents. Premium ice creams are usually higher in fat but also are more flavorful. Try serving puréed strawberries drizzled over ice cream.

- Tuna salad: Tuna in a can is inexpensive and simple to prepare. If sodium is an issue, the tuna can be rinsed. For a quick tuna salad, just open the can, drain, rinse, and mix with mayonnaise and seasonings. Tuna salad can be served with soft crackers.

A healthy diet that tastes good is very important for the frail older adult. Ailments may make it difficult to prepare healthy meals, and snacks become much more important in ensuring optimal nutrient intake. Often, a caregiver is needed to assist with daily tasks like shopping and food preparation. Serve delicious snacks that are easy to prepare and affordable, and that meet the nutritional needs of the frail older adult. Snack time should be enjoyable for both the older adult and the caregiver.

\section{Resources}

Bandinelli, S., and B. Lunenfeld. 2010. "Frailty and the Role of Nutrition in Older People: A Review of the Current Literature." Acta Biomed 81(Suppl. 1): 37-45.

Heuberger, R. A. 2011. "The Frailty Syndrome: A Comprehensive Review." J. Nutr. Gerontol. Geriatr. 30(4): 315-68. 